\title{
REMARK ON INVARIANT MEANS
}

\section{ROBERT KAUFMAN}

In this note $G$ is an abelian group and $m$ is generically an invariant mean in $G$, as defined, for example, in [4]. Probabilistic arguments [Baire's theorem] are applied to the measure [topological] space $2^{G}$ to obtain information about the means $m$. One result, which appears to be new, is an answer to a problem set by R. G. Douglas [2]:

If $2 G$ is infinite, not every invariant mean $m$ is inversion invariant.

The space $2^{G}$. The family $\{S\}$ of subsets of $G$ may be identified in the familiar way with the set of all functions on $G$ to $\{0,1\}$ and provided with the product topology; $2^{G}$ is metrizable if $G$ is countable. Since $\{0,1\}$ is a probability space (the details may safely be suppressed), $2^{G}$ may be provided with the product measure $\mu$, even if $G$ is uncountable; it is sufficient in the present case to regard this measure $\mu$ as a Baire measure in $2^{G}$. For details of the construction see $[3, \S 38]$. As to the way this measure is actually used here, we observe that if $F$ is a subset of $G$ containing exactly $n$ elements, $\mu\{S: F \subseteq S\}$ $=2^{-n}$.

Lемма 1. Let $f$ be $a$ bounded real function on $G$ such that for every finite set $F=\left\{a_{1}, \cdots, a_{n}\right\}$ in $G(n$ may depend on $F)$, sup $\sum_{1}^{n} f\left(x+a_{i}\right)$ $\geqq n$. Then for some invariant mean $m, m(f) \geqq 1$.

Proof. Let $B(G)$ be the Banach space of real bounded functions on $G$ and $B_{0}(G)$ the subspace generated by functions $h_{a}-h$; by definition $h_{a}(x)=h(x+a)$. Let $N$ be the set of nonpositive functions in $B(G)$, and for each $g$ in $B(G)$ define $\omega(g)$ to be the norm-distance of $g$ from the convex set $B_{0}(G)+N$. Then $\omega$ is subadditive and positive-homogeneous, while the argument in $[4, \$ 17.5]$ shows that $\omega(f) \geqq 1$. By the Hahn-Banach theorem there is a linear functional $\lambda$ on $B(G)$ for which $\omega \geqq \lambda$ and $\omega(f) \geqq 1$. Then $\lambda$ is positive, translation invariant and has norm at most 1 as required.

Corollary. If $S$ and $T$ are subsets of $G$, in order that there exist an invariant mean $m$ such that $m(S)=1, m(T)=0$, it is necessary and sufficient that to each finite set $F \subseteq G$ there exist $x$ such that $x+F \subseteq S$, $(x+F) \cap T=\varnothing$. (Here $m(S)=m\left(\xi_{S}\right)$ for $S \subseteq G$.)

Proof. For the necessity, let $F=\left\{a_{1}, \cdots, a_{n}\right\}$ and observe that

Received by the editors November 4, 1965. 
the set $\bigcap_{i=1}^{n}\left(S-a_{i}\right) \sim \cup_{1=1}^{n}\left(T-a_{i}\right)$ has $m$ measure 1 and is thus nonempty. For the sufficiency, apply the previous lemma to the function $f=\xi_{S}-\xi_{T}$, observing that $m(S), m(T) \in[0,1]$.

LemMA 2a. Let $F$ be a finite subset of $G$ and $U=\left\{(S, T) \in 2^{G} \times 2^{a}\right.$ : for some $x, x+F \Phi S,(x+F) \cap T=\varnothing\}$. Then $U$ is an open set; if $G$ is infinite, $U$ is dense and contains an open Baire set of $\mu \times \mu$ measure 1.

Proof. Since $F$ is finite, $U$ is presented as the union of open sets and is thus open. We now assume $G$ is infinite and choose a sequence $\left\{x_{i}: i \geqq 1\right\}$ in $G$ such that $x_{i}-x_{j} \notin F-F$ if $i \neq j$. The complement of $U$ belongs to the closed Baire set

$$
\bigcap_{i=1}^{\infty}\left\{(S, T) \in 2^{G} \times 2^{G}: x_{i}+F \Phi S \text { or }\left(x_{i}+F\right) \cap T \neq \varnothing\right\} .
$$

For each element $x$ of $G$ let $Y_{x}(S, T)$ be the $x$-coordinate of $S$ and $Z_{x}(S, T)$ the $x$-coordinate of $T$. The random variables $Y_{x}, Z_{x}, x \in G$, are jointly independent $[3, \S 45]$ for the measure $\mu \times \mu$, and consequently the sets enclosed in braces are jointly independent for distinct indices $i$. Since each of these sets has the same measure $<1$, the intersection has measure 0 . (If $F$ has $r$ elements, the measure of each set in braces is exactly $1-2^{-2 r}$.) Moreover $U$ is dense, since any open subset in $2^{G} \times 2^{G}$ contains an open Baire set of positive measure.

CoROllaRY. If $G$ is countably infinite, then for almost all pairs $(S, T)$ there is an invariant mean $m$ such that $m(S)=1, m(T)=0$, and an invariant mean $m^{\prime}$ such that $m^{\prime}(T)=1, m^{\prime}(S)=0$. The pairs with this property are a dense $G_{\delta}$.

Proof. Observe that the finite subsets of $G$ may be enumerated and apply Lemma $2 \mathrm{a}$ and the Corollary to Lemma 1.

LEMмA 2b. If $F$ is a finite subset of $G$ then $V=\left\{S \in 2^{a}: x+F \subseteq S\right.$, $(x+F) \cap-S=\varnothing$ for some $x$ in $G\}$ is open. If $2 G$ is infinite, $V$ is dense and contains an open Baire set of $\mu$ measure 1.

Proof. That $V$ is open is clear. If $2 G$ is infinite there is a sequence $\left\{x_{i}: i \geqq 1\right\}$ in $G$ such that $x_{i}+x_{j} \notin F-F$ for all $i, j \geqq 1$ and $x_{i}-x_{j} \notin F$ $-F$ for $i>j \geqq 1$. The complement of $V$ is contained in the closed Baire set

$$
\bigcap_{i=1}^{\infty}\left\{S \in 2^{\sigma}: x_{i}+F \Phi S \text { or }-\left(x_{i}+F\right) \cap S \neq \varnothing\right\} .
$$

The proof now follows that of Lemma 2a. 
Corollary. If $G$ is countably infinite and $2 G$ is infinite, the sets $S \in 2^{G}$ for which $m(S)=1, m(-S)=0$ for some invariant mean $m$ form a dense $G_{\delta}$ of measure 1.

THEOREM 3. If $G$ is infinite, there is more than one invariant mean for $G$. If $2 G$ is infinite, $G$ has invariant means which are not inversion invariant.

Proof. If $G$ is infinite, let $\phi$ be a homomorphism of $G$ on to a countably infinite group. An invariant mean on $\phi(G)$ may be construed as an invariant mean on the field of subsets of $G$ generated by cosets of the kernel of $\phi$. According to $[4, \S 17.14]$ any such set function admits an extension to an invariant mean. Since $\phi(G)$ has many invariant means, so also does $G$. The existence of many invariant means was first proved by Day [1].

If $2 G$ is infinite, let $\psi$ be a homomorphism of $2 G$ onto a countable infinite group, $H$ a countable divisible group containing $\psi(2 G)$ and $\phi$, an extension of $\psi$, which maps $G$ into $H$. Then $\phi(G)$ is countable, $2 \phi(G)=\phi(2 G)$ is infinite. Because $\phi(G)$ has invariant means which are not inversion invariant, so also does $G$, by the argument just stated.

To obtain the converse to the second statement of the theorem, set $f \sim(x)=f(-x)$ and $2 G=\left\{a_{1}, \cdots, a_{n}\right\}, n<\infty$. For a bounded function $f$ and invariant mean $m$

$$
\begin{gathered}
m(f \sim)=m\left(\frac{1}{n} \sum_{i=1}^{n} f_{\tilde{a}_{i}}\right) ; \\
\frac{1}{n} \sum_{i=1}^{n} f_{\tilde{a}_{i}}(x)=\frac{1}{n} \sum_{i=1}^{n} f\left(-x-a_{i}\right) .
\end{gathered}
$$

The identity $-x-2 G=x+2 G$ shows that

$$
\frac{1}{n} \sum_{i=1}^{n} f_{a_{i}}=\frac{1}{n} \sum_{i=1}^{n} f_{a_{i}}
$$

and so $m(f \sim)=m(f)$.

\section{REFERENCES}

1. M. M. Day, Amenable semigroups, Illinois J. Math. 1 (1957), 509-544.

2. R. G. Douglas, On the inversion invariance of invariant means, Proc. Amer. Math. Soc. 16 (1965), 642-644.

3. P. R. Halmos, Measure theory, Van Nostrand, Princeton, N. J., 1950.

4. E. Hewitt and K. A. Ross, Abstract harmonic analysis. I, Springer, Berlin, 1963. 